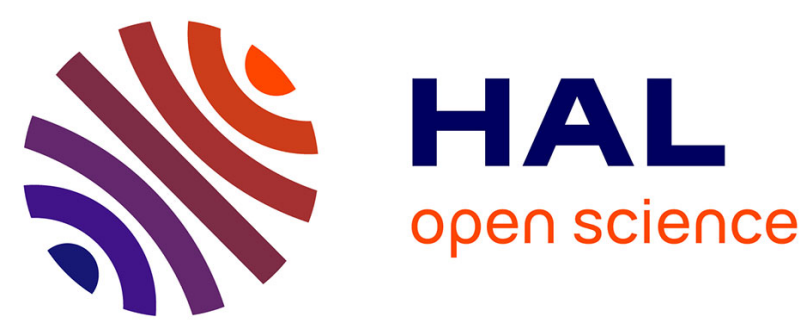

\title{
Compact self-powered synchronous energy extraction circuit design with enhanced performance
}

Weiqun Liu, Caiyou Zhao, Adrien Badel, Fabien Formosa, Qiao Zhu, Guangdi $\mathrm{Hu}$

\section{- To cite this version:}

Weiqun Liu, Caiyou Zhao, Adrien Badel, Fabien Formosa, Qiao Zhu, et al.. Compact self-powered synchronous energy extraction circuit design with enhanced performance. Smart Materials and Structures, 2018, 27 (4), 10.1088/1361-665x/aab339 . hal-01901539

\section{HAL Id: hal-01901539 \\ https://hal.science/hal-01901539}

Submitted on 23 Oct 2018

HAL is a multi-disciplinary open access archive for the deposit and dissemination of scientific research documents, whether they are published or not. The documents may come from teaching and research institutions in France or abroad, or from public or private research centers.
L'archive ouverte pluridisciplinaire HAL, est destinée au dépôt et à la diffusion de documents scientifiques de niveau recherche, publiés ou non, émanant des établissements d'enseignement et de recherche français ou étrangers, des laboratoires publics ou privés. 


\title{
Compact self-powered synchronous energy extraction circuit
}

\section{design with enhanced performance}

\author{
Weiqun Liu ${ }^{1}$, Caiyou Zhao ${ }^{2}$, Adrien Badel ${ }^{3}$, Fabien Formosa ${ }^{3}$, Qiao Zhu ${ }^{1}$, Guangdi Hu ${ }^{1}$ \\ ${ }^{1}$ School of Mechanical Engineering, Southwest Jiaotong University, 610031 Chengdu, China \\ ${ }^{2}$ School of Civil Engineering, Southwest Jiaotong University, 610031 Chengdu, China \\ ${ }^{3}$ Laboratoire Systèmes et Matériaux pour la Mecatronique, Université Savoie Mont-Blanc, 7 \\ chemin de Bellevue, 74944 Annecy le Vieux, France \\ Email: zcy848279@163.com
}

\begin{abstract}
Synchronous switching circuit is viewed as an effective solution of enhancing the generator's performance and providing better adaptability for load variations. A critical issue for these synchronous switching circuits is the self-powered realization. In contrast with other methods, the electronic breaker possesses the advantage of simplicity and reliability. However, beside the energy consumption of the electronic breakers, the parasitic capacitance decreases the available piezoelectric voltage. In this technical note, a new compact design of the self-powered switching circuit using electronic breaker is proposed. The envelope diodes are excluded and only a single envelope capacitor is used. The parasitic capacitance is reduced to half with boosted performance while the components are reduced with cost saved.
\end{abstract}

Keywords: Energy harvesting Piezoelectric generator Synchronous switching circuit Electronic breaker

\section{1- Introduction}

With the growth of electronic portable devices, autonomous energy systems and autonomous network systems, devices which can generate electrical power by exploiting ambient vibration energy have been developed in recent years. In addition to the improvement of the power density [1] or the bandwidth [2-5] of the generator through the mechanical structure, the interface circuit plays a critical role in the harvester's performance, especially for the piezoelectric generator with high output impedance. Dedicated circuit design is required to extract more power from the generator and improve the impedance match. In comparison with the usual standard circuit of a rectifier and a filter capacitor [6], the synchronous switching circuit is viewed as an effective solution of enhancing the generator's performance and providing better adaptability for load variations. The SSHI (Synchronous Switching Harvesting on Inductor) [7] is firstly introduced to utilize the voltage inversion process to avoid the energy return 
phenomena in the standard circuit. A diversity of improved circuits based on SSHI are then proposed, such as SSHI-MR [8], DSSH [9], ESSH [10] and so on. In addition, the SECE (Synchronous Electric Charge Extraction) [11] is raised to solve the impedance matching problem. Furthermore, the OSECE (Optimized SECE) is promoted to improve the performance of SECE while keeping low load dependence [12] while the tuneable SECE is developed to extend the bandwidth of generator from the circuit part [13]. However, a critical issue for these synchronous switching circuits is the self-powered realization.

Various methods including mechanical switches [14-17], velocity control [18], integrated control circuits [19-20], electronic breakers [21-25] etc. have been developed. Among them, the mechanical switches approach has the fewest electronic components with the contacting electrodes [14-16] or the reed switch [17] instead of the electronic switches. However, it is not so adaptive to the displacement variations in many cases. The velocity control utilizes an additional sensing unit to detect the displacement extreme and control the switches precisely with analogue comparators [18]. Additional fabrication requirements are usually required for the piezoelectric generator. The integrated control circuits [19-20] have the strongest function but also the most components and the most complicated design. In contrast, the electronic breaker possesses the advantage of simplicity and reliability. Generally, a part of the extracted energy from the generator is consumed by the electronic breaker and the switching phase lag is introduced by the envelope detector [21-23] and the charging of the switches [25]. Moreover, the parasitic capacitance in the electronic breaker, mainly referred to the envelope detector capacitance, imposes an equivalent effect of connecting a parallel capacitor of the total parasitic capacitance to the generator and induces the charge neutralization after the voltage inversion, thus decreasing the available piezoelectric voltage, especially for the generators with small intrinsic capacitance. Consequently, the generator's performance degenerates obviously compared with the ideal case.

Reducing the components of the self-powered circuit components while keeping the function could save the energy consumption and would be helpful for saving the costs. Recently, an alternative SSHI self-powered design using electronic breakers has been proposed with fewer diodes used and better performance [26]. However, two capacitors are still used in the circuit for peak detectors. In comparison with the diodes, the capacitance plays a more critical role in the performance by introducing the switching phase lag and decreasing the piezoelectric voltage. In this technical note, a CSPOSECE (Compact SelfPowered OSECE) design is propose by multiplexing the comparator instead of the diode in the regular SPOSECE circuit [23]. More important, only a single capacitor is used for both positive and negative peak detection. The parasitic capacitance of the electronic breaker is thus reduced to half so that the performance is improved. 


\section{2- Proposed CSP-OSECE circuit}

\section{1- Circuit principle}

Fig. 1 (a) shows the SP-OSECE circuit with both NPN and PNP power transistors as the switches, which are different from previous works with both NMOS switches [23]. The circuit mainly consists of two electronic breakers, two transistor switches, a three-winding fly-back transformer (turn ratio $L_{1}: L_{2}: L_{3}=$ 1:1:m) and the load unit. The positive and negative breakers are connected to the two windings of the transformer's primary side respectively while the secondary side is connected to the load unit. Each electronic breaker is composed of an envelope detector and a comparator. In the circuit, the piezo element is represented by an equivalent current source $i_{e q}$ and an intrinsic capacitance $C_{0}$. When the piezoelectric element is subjected to a sinusoidal excitation with the displacement $u=u_{M} \sin \omega t$, we have

$$
i_{e q}=\alpha \dot{u}=\alpha \omega u_{M} \cos \omega t
$$

in which $\alpha$ is the piezoelectric force factor, $\omega$ is the excitation frequency and $u_{M}$ is the displacement amplitude.

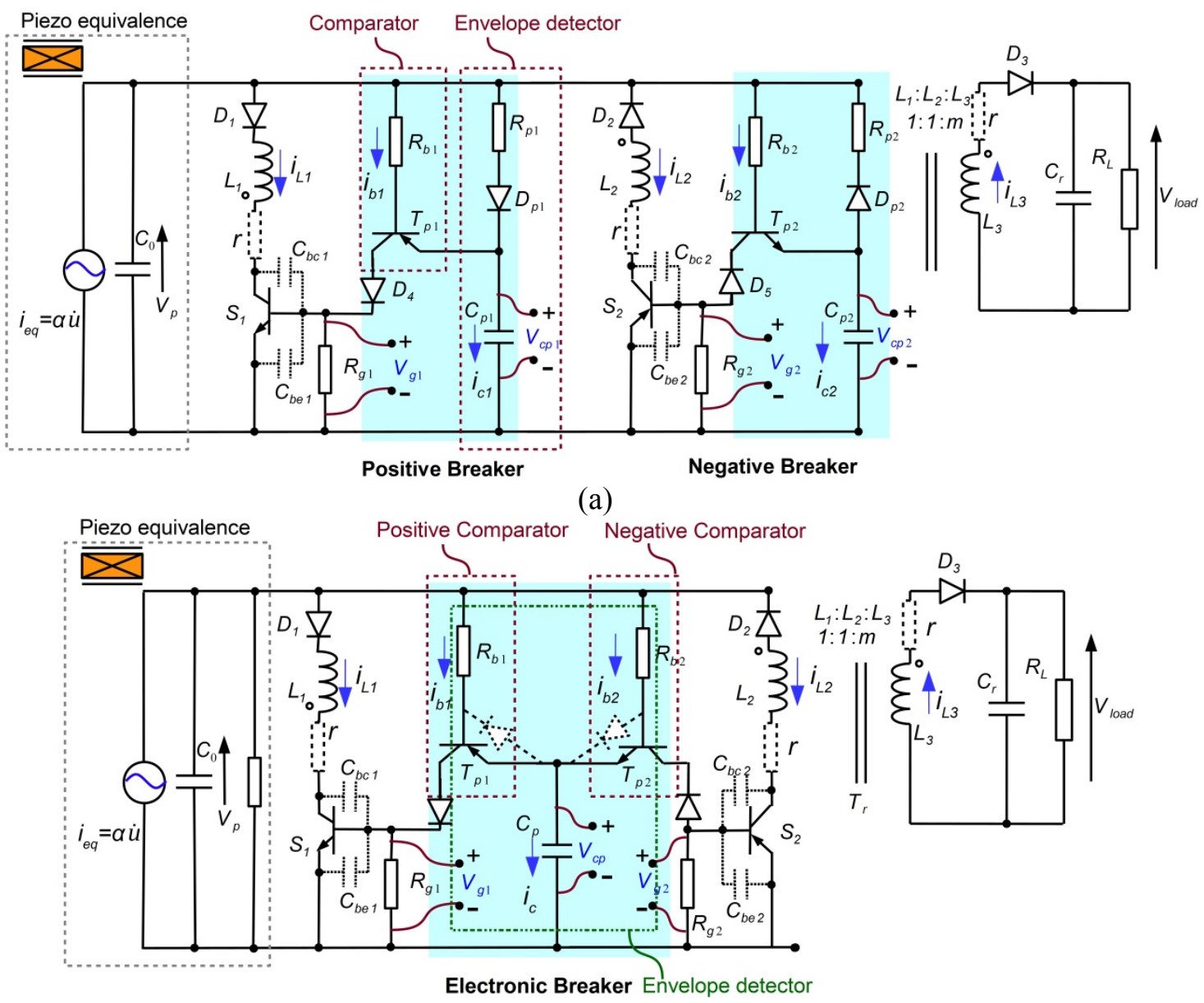

(b)

Fig. 1. Self-powered realizations of the OSECE circuit: (a) SP-OSECE; (b) CSP-OSECE. 

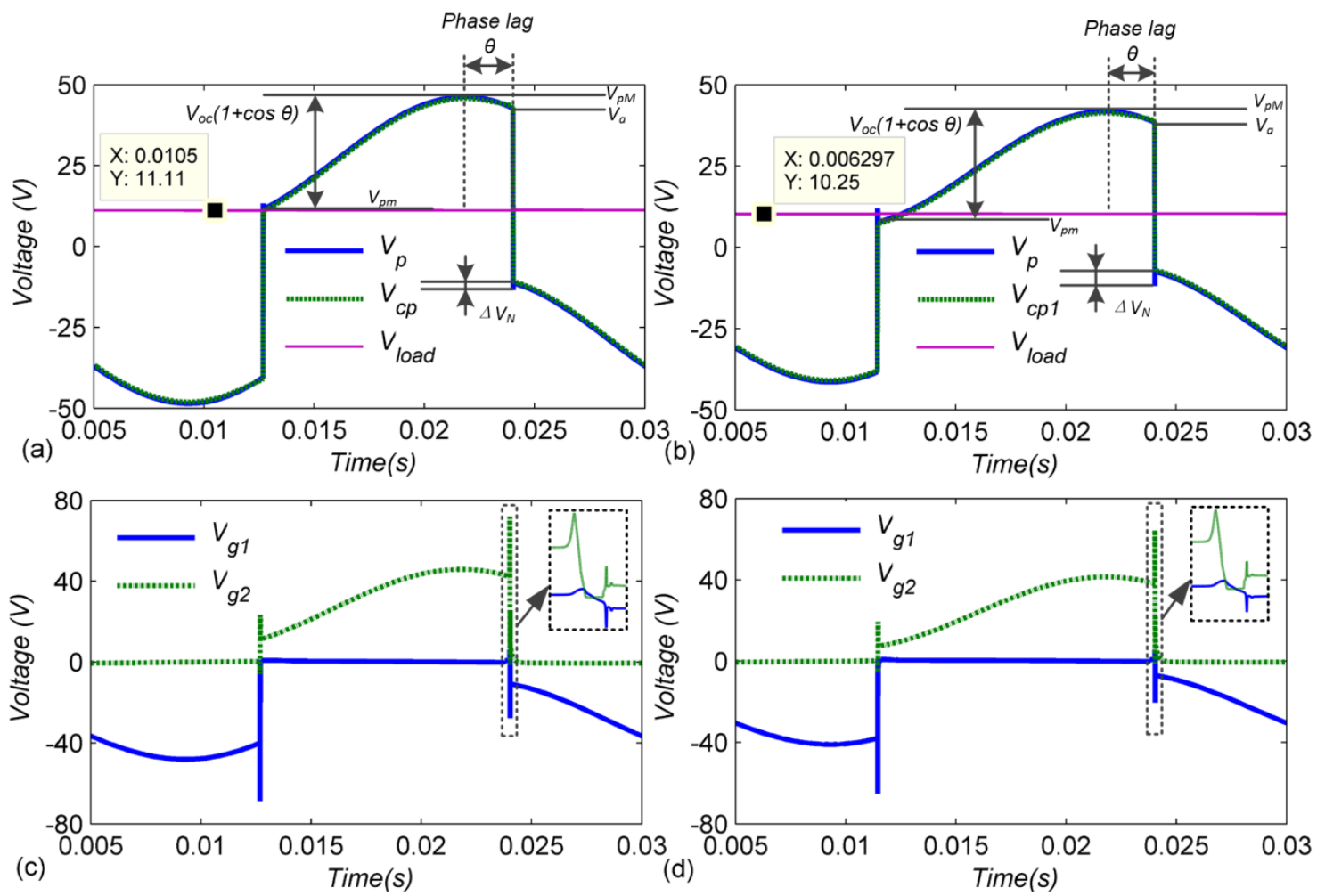

Fig. 2. Simulated Waveforms of the CSP-OSECE and SP-OSECE circuits: (a) and (c) for CSP-OSECE, (b) and (d) for SP-OSCE.

The operation principle is simply introduced here for easy readability and more details can be found in $[23,25]$. In the positive half-period, $C_{0}$ is charged by the current source $i_{e q}$ and the piezoelectric voltage $V_{p}$ increases until the peak $V_{p M}$. With this peak value reserved on the envelope capacitance $C_{p 1}$ in the form of $V_{c p 1}, V_{p}$ begins to decrease due to the reverse charging by $i_{e q}$. As $V_{p}$ is lower than $V_{c p 1}$ with the difference of $V_{\mathrm{be}}$, leading to the conduction of the comparator $T_{p 1}$, the current from $C_{p 1}$ starts to charge the baseemitter parasitic capacitance $C_{b e 1}$ to turn on the switch $S_{1}$. Afterwards, a LC oscillation $\left(L_{1}\right.$ and $\left.C_{0}\right)$ begins and the piezoelectric voltage is inverted. As soon as $V_{p}$ reaches $-V_{\text {load }} / m$, the remained energy in the transformer is transferred to the load due to the special mechanism of the fly-back transformer. Subsequently, the negative half-period starts with similar operations. In this regular SP-OSECE circuit of Fig. 1 (a), the positive breaker and the negative breaker are independent to each other with similar yet complementary configurations. Two separate sets of envelope detectors are used, including the diodes $\left(D_{p 1}\right.$ and $\left.D_{p 2}\right)$, the resistors $\left(R_{p 1}\right.$ and $\left.R_{p 2}\right)$ and the capacitors $\left(C_{p 1}\right.$ and $\left.C_{p 2}\right)$.

Fig. 1 (b) presents the proposed CSP-OSECE (Compact SP-OSECE) design. The improvements in comparison with the regular SP-OSECE circuit arise from two aspects: (1) the comparators $T_{p i}$ are multiplexed as the envelope diodes which are indicated by the dashed symbols in Fig. 1 (b); (2) a single envelope capacitor is shared by both positive and negative breakers. In the positive half-period, $R_{b 2}$, the base-emitter PN junction of $T_{P 2}$ and $C_{p}$ work as the positive envelope detector; in the negative half-period, 
$R_{b 1}$, the base-emitter PN junction of $T_{p 1}$ and $C_{p}$ work as the negative envelope detector. The circuit operations are similar to the regular SP-OSECE circuit and the simulated voltage waveforms for both circuits are plotted in Fig. 2 with $C_{p}=1.75 \mathrm{nF}$ and $R_{\text {load }}=470 \mathrm{k} \Omega$ while other components and parameters are listed in Table 1. Notably, the variables are denoted in Fig. 1. Easy to find, the produced switching control signals $V_{g 1}$ and $V_{g 2}$ are almost the same while the piezoelectric voltage $\left(V_{\mathrm{p}}\right)$ waveforms are similar as well.

Replacing the rectifying diode with the PN junction inside the bipolar transistor comparator might require special attention to the interchangeability. By observing the circuit in Fig. 1 and the waveforms in Fig.2, it can be found that the voltage on the envelope capacitor $V_{c p}$ always follows the piezoelectric voltage with a voltage difference of $+/-V_{D}\left(V_{B E}\right)$. It means that the voltage stress on the PN junction is small. Therefore, the only issue is the current rating of the PN junction. Considering that the current from the piezoelectric element to the envelope capacitor is usually small in micro-power energy harvesting cases, few efforts are added when selecting the components for the comparator function. However, it should be noticed that the voltage drop on the PN junction might be higher than the rectifier diode. Normally, the voltage difference is small and negligible but it might bring some performance degeneration by introducing additional phase lag, especially for the low piezoelectric voltage case. Therefore, it is recommended to select the bipolar transistor with small $V_{B E}$ value.

Clearly, the required components in CSP-OSECE circuit are less than the SP-OSECE circuit with the same function. The needs for specific envelope resistors and diodes are excluded. More important, the parasitic capacitance in the circuit is reduced to a single capacitor. It brings additional performance enhancement which will be detailed in next section.

However, it has to be pointed out that the proposed CSP-OSECE circuit is only applicable to the case of complementary branches with $\mathrm{N}$ type switch for positive breaker and $\mathrm{P}$ type switch for negative breaker. In addition, the bipolar transistors have to be used as the comparators due to the multipurpose use. When the MOSFET transistor or the nano-power integrated comparator such as LT1540 is used [27], the idea of multipurpose use in CSP-OSECE circuit is not suitable.

\section{2- Performance analysis}

For the OSECE approach, the harvested power after stabilization can be expressed as

$$
P=\frac{V_{\text {load }}^{2}}{R_{L}}=2 f \times \Delta E \approx 2 f \times \frac{1}{2} \eta C_{0}\left(V_{a}^{2}-\frac{V_{\text {load }}^{2}}{m^{2}}\right)
$$

in which $f=\omega /(2 \pi)$ is the excitation frequency, $\Delta E$ is the transferred energy during each switching operation, $\eta$ is the energy transfer efficiency related to the circuit quality factor and $V_{a}$ is the piezoelectric voltage at the instant right before the switching operation. And we have 


$$
V_{a} \approx V_{p m}+2 V_{o c} \cos \theta \approx \frac{V_{\text {load }}}{m}-\Delta V_{N}+2 V_{o c} \cos \theta
$$

Here, $V_{p m}$ is the local minimum of $V_{p}$ after the voltage inversion, $V_{o c}$ is the open-circuit voltage magnitude of the piezo element when connecting to the extraction circuit with the switches turn-off and $\theta$ is the switching phase lag related to piezoelectric maximum $V_{p M}$. It is noted that $V_{p m}$ is smaller than $V_{\text {load }} / m$ with a difference of about $\Delta V_{N}$ which refers to the voltage retreatment towards zero after the voltage inversion as seen in Fig. 2. This voltage retreat phenomena is due to the charge neutralization of the parasitic capacitance in the circuit, mainly relates to the envelope detector capacitor $C_{p i}$, as pointed out in [22, 25].

Using eq. (2) and eq. (3), the power can be written in the other form as

$$
P=\frac{V_{\text {load }}^{2}}{R_{L}} \approx \frac{m^{2} \eta f C_{0}\left(-\Delta V_{N}+2 V_{o c} \cos \theta\right)^{2}}{\left(\sqrt{m^{2}+\eta f C_{0} R_{L}}-\sqrt{\eta f C_{0} R_{L}}\right)^{2}}
$$

With the same switches, load resistor and transformer used for the regular SP-OSECE and the CSPOSECE, the values of $C_{0}, R_{L}$ and $m$ are identical for both circuits. Moreover, when the envelope resistor $R_{p i}$ has the same value of $R_{b i}$ and the voltage drop on the diode $D_{p i}$ in SP-OSECE are close to the drop on $T_{p i}$ in CSP-OSECE, the phase lag $\theta$ is inclined to have approximate values as well in both cases since the factors inducing the phase lag are almost the same. It is partly verified by the waveforms in Fig. 2 (a) and (b) that the approximate phase lag values are obtained for both cases. Furthermore, it can be inferred that the values of the efficiency $\eta$ are expected to be similar with the above conditions since the three circuit branches ((1) $L_{1}, S_{1}, D_{1}$; (2) $L_{2}, S_{2}, D_{2}$ and (3) $L_{3}, D_{3}$ ) related to the voltage inversion process are the same in both circuits. Consequently, the power difference between the regular SP-OSECE and the CSP-OSECE are mainly related to the open-circuit voltage magnitude $V_{o c}$ and the voltage retreatment $\Delta V_{N}$ due to the charge neutralization. Therefore, comparative investigations are further performed on these two factors.

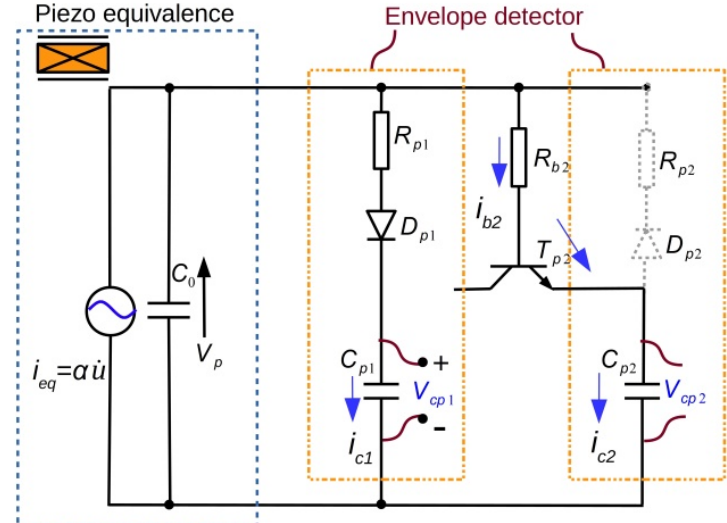

(a)

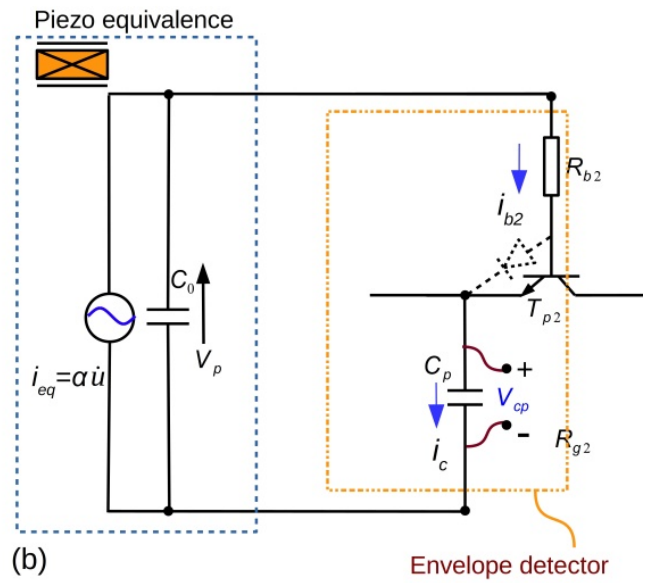

(b)
Envelope detector

Fig. 3 Equivalent circuit of the two circuits in the charging phase: (a) CSP-OSECE; (b) SP-OSECE. 
According to the definition, the open-circuit voltage magnitude $V_{o c}$ can be obtained with the assumption that the switches $S_{1}$ and $S_{2}$ are turned off. It is similar to the charging phase between two switching operations. By carefully observing the regular SP-OSECE circuit in Fig. 1 (a) and the CSPOSECE circuit in Fig. 1 (b) in this case, they can be simplified to the reduced circuits in Fig. 3 (a) and (b) respectively. Here, only the positive half-period is considered while the negative half-period can be obtained in the same way. Clearly, beside $C_{0}$, the equivalent current source $i_{e q}$ is required to charge $C_{p 1}$ through $R_{p 1}, D_{p 1}$ and $C_{p 2}$ through $R_{p 2}, T_{p 2}$ simultaneously. When $\omega R_{p i} C_{p i}<<1$ and $\omega R_{b i} C_{p i}<<1$ are satisfied for the usual case, the open-circuit voltage magnitude $V_{o c}$ can be written as

$$
V_{o c @ S P_{-} O S E C E} \approx \frac{\alpha u_{M}}{C_{0}+2 C_{p}}=\frac{C_{0} V_{o c, o r g}}{C_{0}+2 C_{p}}
$$

for the regular SP-OSECE circuit. Here, $C_{p 1}=C_{p 2}=C_{p}$ and $V_{o c, o r g}=\alpha u_{M} / C_{0}$ is the original open-circuit voltage of the piezoelectric generator without any circuits. In contrast, $i_{e q}$ only needs to charge a single capacitor $C_{p}$ beside $C_{0}$ in the CSP-OSECE circuit and we have:

$$
V_{o c @ C S P \_O S E C E} \approx \frac{\alpha u_{M}}{C_{0}+C_{p}}=\frac{C_{0} V_{o c, o r g}}{C_{0}+C_{p}}
$$

Easy to find, a higher open-circuit voltage magnitude is obtained for CSP-OSECE in comparison with the regular SP-OSECE and the difference increases with $C_{p}$.

The charge neutralization refers to the short phase after the voltage inversion during which the charge on $C_{0}$ is redistributed on $C_{0}, C_{p 1}$ and $C_{p 2}$ for SP-OSECE or $C_{0}$ and $C_{p}$ for CSP-OSECE until new voltage equilibrium between them. It induces the voltage retreatment towards zero and we have

$$
\Delta V_{N @ S P-O S E C E} \approx \frac{V_{\text {load }}}{m}-\frac{C_{0} V_{\text {load }} / m-q_{r 1}-q_{r 2}}{C_{0}+2 C_{p}}
$$

for SP-OSECE and

$$
\Delta V_{N @ C S P-O S E C E} \approx \frac{V_{\text {load }}}{m}-\frac{C_{0} V_{\text {load }} / m-q_{r 0}}{C_{0}+C_{p}}
$$

for CSP-OSECE. Here, $q_{r 1} \approx C_{p} V_{a} \approx C_{p}\left(V_{\text {load }} / m+2 V_{o c} \cos \theta\right)$ and $q_{r 2} \approx C_{p}\left(V_{b e}+V_{D}+V_{c e}\right)$ are the remained charge on $C_{p 1}$ and $C_{p 2}$ to be neutralized respectively in the SP-OSECE circuit in Fig. 1 (a) and $q_{r 0} \approx C_{p}\left(V_{b e}+V_{D}+V_{c e}\right)$ is the remained charge on $C_{p}$ to be neutralized in the CSP-OSECE circuit. $V_{b e}, V_{D}$ and $V_{c e}$ represent the base-emitter voltage drop of the switches $S_{i}$, the voltage drop on the diodes $D_{i}$ and the voltage collector- 
emitter voltage drop of the comparators $T_{p i}$ respectively. Considering $q_{r 2}<<q_{r 1}$ and $q_{r 0}<<C_{0} V_{\text {load }} / m$, eq. (7) and eq. (8) can be further simplified as:

$$
\begin{gathered}
\Delta V_{N @ S P-O S E C E} \approx \frac{3 C_{p} V_{\text {load }} / m+2 C_{p} V_{o c} \cos \theta}{C_{0}+2 C_{p}} \\
\Delta V_{N @ C S P-O S E C E} \approx \frac{C_{p} V_{\text {load }} / m}{C_{0}+C_{p}}
\end{gathered}
$$

Clearly, the CSP-OSECE has smaller $\Delta V_{N}$ values than the SP-OSECE circuit does and the difference increases with $C_{p}$ as well.

Combining eq. (5), eq. (6), eq. (9), eq. (10) and using eq. (4), we have the following equation

$$
\frac{P_{S P-O S E C E}}{P_{C S P-O S E C E}}=\frac{\left(2 V_{o c @ S P-O S E C E} \cos \theta-\Delta V_{N @ S P-O S E C E}\right)^{2}}{\left(2 V_{o c @ C S P-O S E C E} \cos \theta-\Delta V_{N @ C S P-O S E C E}\right)}
$$

in which $P_{S P-O S E C E}$ is the power of the SP-OSECE circuit and $P_{C S P-O S E C E}$ is the power of the CSP-OSECE circuit. Since $V_{o c @ S P-O S E C E}<V_{o c @ C S P-O S E C E}$ and $\Delta V_{N @ S P-O S E C E}>\Delta V_{N @ C S P-O S E C E}$ are satisfied, the power performance of CSP-OSECE is always superior to the one of SP-OSECE. Moreover, it can be inferred that the advantages of the CSP-OSECE is especially obvious for large $C_{p}$ values.

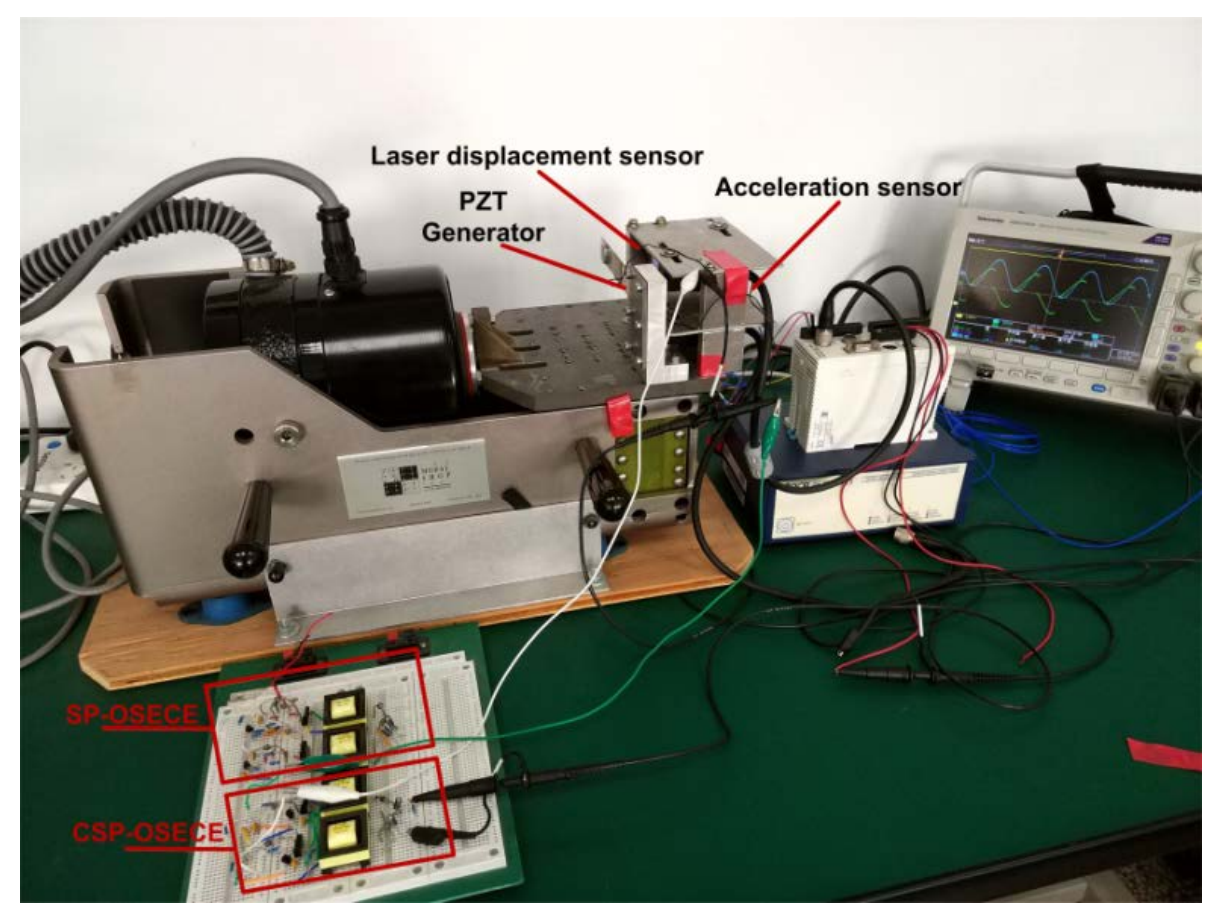

Fig. 4. Experimental platform. 


\section{3- Experimental platform}

Fig. 4 presents the experimental set-up for the validation of the CSP-OSECE circuit's performance. A piezoelectric cantilever generator is horizontally placed on the shaker (2075E-HT, The Modal Shop(C) with a fixture. Two piezoelectric patches $(20 \mathrm{~mm} \times 10 \mathrm{~mm} \times 0.4 \mathrm{~mm})$ are attached to the top and bottom of the steel cantilever $(20 \mathrm{~mm} \times 10 \mathrm{~mm} \times 0.6 \mathrm{~mm})$ respectively. A small mass of 5.7 gram is fixed on the beam tip to pick up the excitation. When the shaker is driven by a signal source (DG1032, RigolC), an acceleration sensor $\left(\mathrm{M} 352 \mathrm{C} 68, \mathrm{PCB}^{\circ}\right)$ is used to measure the excitation magnitude and a laser displacement sensor (HL-C203BE, SUNXC) is adopted to acquire the vibration responses of the generator. Both CSP-OSECE and SP-OSECE appoaches are elaborated with the same compents listed in table 1 except the different ciruit configurations. The piezoelectric generator is connected to these two circuit alternatively under the same conditions for fair comparison. The load voltage is then sampled by an oscilloscope together with the acceleration and the displacment signals. Parameters identification is firstly performed to the generator with the values given in table 1 . For each piezoelectric patch, $\alpha$ and $C_{0}$ are estimated to $0.0003 \mathrm{NV}^{-1}$ and $14 \mathrm{nF}$ respectively. Meanwhile, the open-circuit resonant frequency is found to be around $41 \mathrm{~Hz}$. Since the energy transfer efficiency $\eta$ is related to the circuit working condition and the load voltage [24], it fluctuates in a certain range from around $40 \%$. For simplification, a constant efficiency is assumed for theoretical modeling with this value.

Table 1. Components and Parameters

\begin{tabular}{cccc}
\hline Definition & Value & Definition & Value \\
\hline Switches $\left(S_{i}\right)$ & TIP31C & Transistor $\left(T_{p i}\right)$ & $2 \mathrm{~N} 5401$ \\
Transformer $T_{1}$ & WE750811290 $(m=1)$ & $C_{0}$ (single piezo) & $14 \mathrm{nF}$ \\
Diodes $\left(D_{i}, D_{p i}\right)$ & BYV28-100 & $C_{0}$ (two piezo) & $28 \mathrm{nF}$ \\
$f$ & $41 \mathrm{~Hz}$ & $R_{b i}$ & $3.3 \mathrm{k} \Omega$ \\
$R_{g i}$ & $1 \mathrm{M} \Omega$ & $R_{p i}$ & $3.3 \mathrm{k} \Omega$ \\
$u_{M}$ & $1.25 \mathrm{~mm}$ & $\alpha($ single piezo) & $0.0003 \mathrm{~N} / \mathrm{V}$ \\
\hline
\end{tabular}

\section{4- Results}

In order to focus on the circuit performance, the constant displacement case is firstly studied here as the main part. In this case, the displacement of the beam is controlled to be constant with $u_{M}=1.25 \mathrm{~mm}$ and the piezoelectric generator is considered as an equivalent current source $i_{e q}$. As analyzed before, the circuit performance is closely related to the envelope capacitance $C_{p}$. Therefore, tests are repeated for both 
circuits with $C_{p}$ varying from $0.5 \mathrm{nF}$ to $8.2 \mathrm{nF}$ and the load resistor is fixed to $470 \mathrm{k} \Omega$. The results are presented in Fig. 5 for two situations of a single piezoelectric patch or two patches.

In both situations, the harvested power firstly increases and then decreases for two circuits. The reason has been explained in [25] that, small $C_{p}$ values are not able to make the switch in the optimal working condition due the large phase lag and conduction loss while large $C_{p}$ values decrease the available piezoelectric voltage. Therefore, an optimal $C_{p}$ exists for the self-powered switching control circuits with electronic breakers as seen in Fig. 5. It is worthy of note that the CSP-OSECE circuit is superior to the SPOSECE one with better power results in both $C_{0}$ cases. When a single piezoelectric patch $\left(C_{0}=14 \mathrm{nF}\right)$ is used, a maximum power of $0.281 \mathrm{~mW}$ is obtained for the CSP-OSECE in comparison with the value of $0.204 \mathrm{~mW}$ for the regular SP-OSECE at the optimal $C_{\mathrm{p}}$ values which are around $1.75 \mathrm{nF}$. A boost of $37.8 \%$ is found. For the case of two piezoelectric patches, the maximum power of the CSP-OSECE circuit is $0.697 \mathrm{~mW}$ which is about $40.8 \%$ higher than the SP-OSECE's value of $0.495 \mathrm{~mW}$ and the optimal $C_{p}$ values are around 3nF. Due to the CSP-OSECE's improvement of less parasitic capacitance with a single envelope capacitor used, the circuit performance is obviously enhanced. Moreover, it is found that the power enhancement is more obvious as $C_{p}$ increases. It is in accordance with the analysis in section 2 . The theoretical results are also plotted in Fig. 5. Good agreements between experimental and theoretical results are found.

The performance comparison for different load resistance is also performed for both circuits with the estimated optimal $C_{p}$ used $\left(C_{p}=1.75 \mathrm{nF}\right.$ for $C_{0}=14 \mathrm{nF} ; C_{p}=3 \mathrm{nF}$ for $\left.C_{0}=28 \mathrm{nF}\right)$. Cleary, the CSP-OSECE circuit outperforms the regular SP-OSECE circuit for the whole load range in both $C_{0}$ cases. For the case of $C_{0}=14 \mathrm{nF}$, the maximum power of the CSP-OSECE circuit is $0.301 \mathrm{~mW}$ with $R_{\text {load }}=670 \mathrm{k} \Omega$ where the maximum power of the regular SP-OSECE is $0.23 \mathrm{~mW}$. For the case of $C_{0}=28 \mathrm{nF}$, the maximum power values of $0.697 \mathrm{~mW}$ and $0.495 \mathrm{~mW}$ are obtained with $R_{\text {load }}=470 \mathrm{k} \Omega$. Good agreements are still found between theory and experiment except the discrepancy for large load resistance. It is because the efficiency decreases in these cases due to longer switching duration and larger consumption on switches.

Clearly, better performance is always seen by the CSP-OSECE circuit due to the less parasitic capacitance and charge neutralization for the constant displacement case. It means that more damping effect on the generator will be exerted by the CSP-OSECE circuit as well. However, for the constant force case, the optimal power performance is obtained when the damping effect induced by the harvesting process matches the mechanical damping [12]. When the damping effect induced by the harvesting process is higher than the mechanical damping, the performance decreases. The CSP-OSECE with higher damping effect would be not as good as the SP-OSECE. Generally, the relationship between the damping induced by the harvesting process and the mechanical damping can be roughly indicated by a specific 
value of $k^{2} Q_{m}$ for different extraction circuits. Here, $k^{2}$ means the electromechanical coupling level while $Q_{m}$ refers to the mechanical quality factor. For the ideal OSECE approach, this value of $k^{2} Q_{m}$ is around $\pi / 4$ corresponding to the case where the damping induced by the harvesting process is optimal. Since the selfpowered OSECE approaches are affected by many parasitic effects, such as the phase lag of the switching, it is difficult to obtain a determined value. However, studies show that higher values are expected in comparison with the ideal one [23]. Therefore, it can be inferred that for the generators with $k^{2} Q_{m}<\pi / 4$, the CSP-OSECE circuit would achieve better performance than the SP-OSECE one. As a complement, comparative experimental investigations about these two circuits are also performed for the constant acceleration case with a single piezoelectric patch used. With $k^{2} Q_{m}=0.145$, it hints that either CSP-OSECE or SP-OSECE imposes less damping effect than the mechanical damping for the studied cases. Fig. 7 presents the obtained power results of these two circuits. Obvious power improvement is still observed for the CSP-OSECE circuit as expected by the analysis. A power boost of $38 \%$ is found around the resonant frequency.
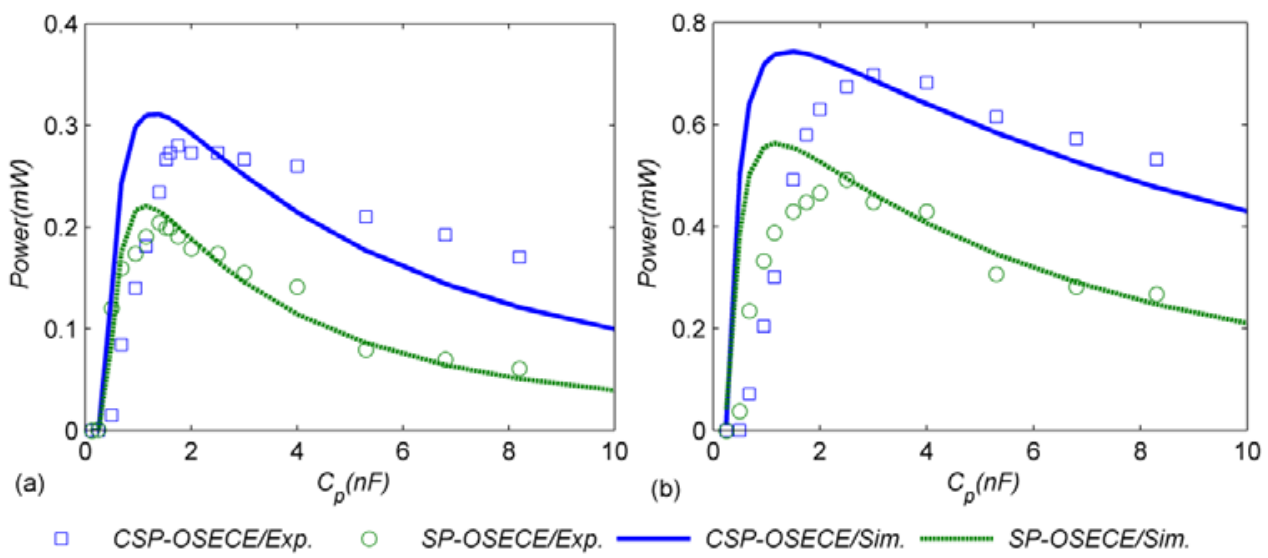

Fig. 5. Power results of both circuits with various $C_{p}$ and constant $R_{\text {load }}$ (a) a single piezoelectric patch $\left(C_{0}=14 \mathrm{nF}\right)$; (b) two piezoelectric patches $\left(C_{0}=28 \mathrm{nF}\right)$.
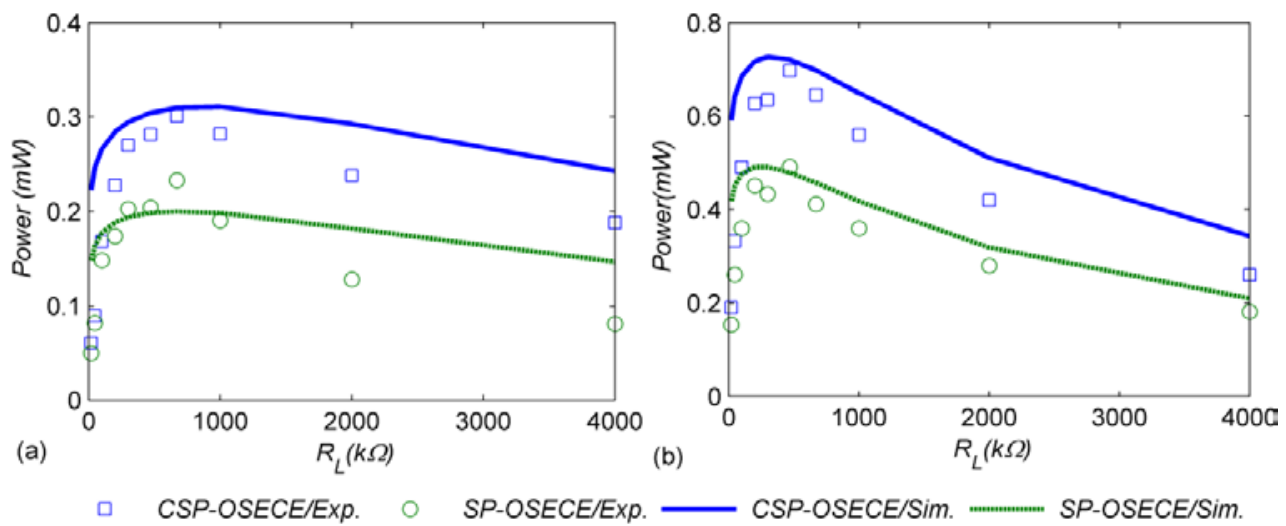

Fig. 6. Power results of both circuits with various $R_{\text {load }}$ and constant $C_{p}$ : (a) a single piezoelectric patch $\left(C_{0}=14 \mathrm{nF}\right) ;(\mathrm{b})$ two piezoelectric patches $\left(C_{0}=28 \mathrm{nF}\right)$. 


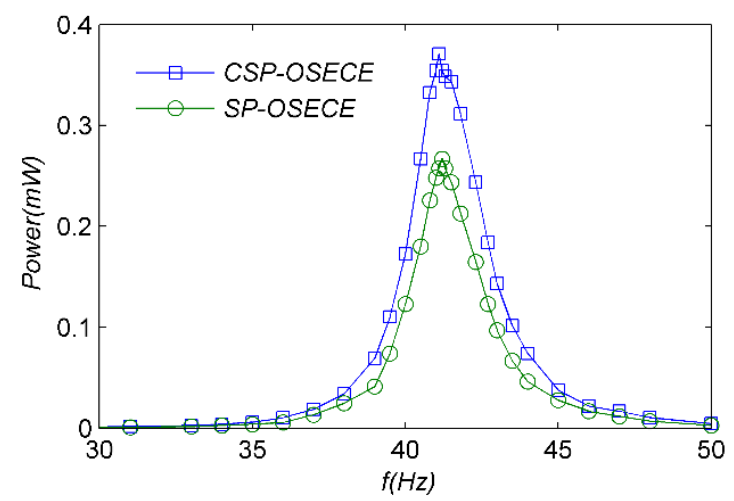

Fig. 7. Experimental power results comparison between CSP-OSECE and SP-OSECE for the constant acceleration of $5 \mathrm{~m} \mathrm{~s}^{-2}$ with a single piezoelectric patch used $\left(R_{\text {load }}=470 \mathrm{k} \Omega\right.$ and $\left.C_{p}=1.75 \mathrm{nF}\right)$.

\section{5- Theoretical discussion}

In order to obtain a more complete performance comparison between the two circuits, further theoretical investigations are conducted on the two circuits. Two relevant factors are considered: the original open-circuit voltage magnitude $V_{o c, o r g}$ and the intrinsic capacitance $C_{0}$. For simplicity, the load is assumed to be a constant value of $100 \mathrm{k} \Omega$ in both cases while other parameters are adopted from Table 1 .

The generators with $C_{0}$ values from $10 \mathrm{nF}$ to $50 \mathrm{nF}$ are firstly investigated with $V_{o c, o r g}=25 \mathrm{~V}$. Fig. 8 (a) presents the power results of the CSP-OSECE and the SP-OSECE approaches for different $C_{0}$ and $C_{p}$ while Fig. 8(b) shows the performance improvement of the CSP-OSECE in comparison with the regular SP-OSECE. Clearly, the performance improvement is most obvious for small $C_{0}$ and large $C_{\mathrm{p}}$ cases in which $C_{p}$ has more influence on $V_{o c}$ and $\Delta V_{N}$, and it is not so significant for large $C_{0}$ and small $C_{p}$ cases in which $C_{p}$ has weaker influence. Considering that the max power at the optimal $C_{p}$ value for each $C_{0}$ case is more relevant, the enhancement in these points is also plotted in Fig. 9. It is found that the max power enhancement decreases as $C_{0}$ increases. The reason is that the ratio $C_{p} / C_{0}$ gets smaller due the slow increase of the optimal $C_{p}$ as seen in Fig. 8 (a). Consequently, the effects of $C_{p}$ on the harvested power become less important so that the performance improvement by CSP-OSECE is less significant as well. However, for the case of $C_{0}=50 \mathrm{nF}$, the power boost still reaches $19.4 \%$.

The influence of the original open-circuit voltage magnitude $V_{o c, o r g}$ is also studied for the generator with $C_{0}=14 \mathrm{nF}$. Considering that the optimal $C_{p}$ corresponding to a fixed $C_{0}$ does not vary much when $V_{o c, o r g}$ changes, $C_{p}$ is fixed to $1.75 \mathrm{nF}$ in this case. Fig. 10 presents the power increase with $V_{o c, o r g}$ changing from $15 \mathrm{~V}$ to $45 \mathrm{~V}$. The power improvement slightly decrease to an almost stable value as $V_{o c, \text { org }}$ increases. The possible reason is that the parasitic capacitance plays a more critical role in the case of low opencircuit voltage magnitude close to the circuit's start voltage. 

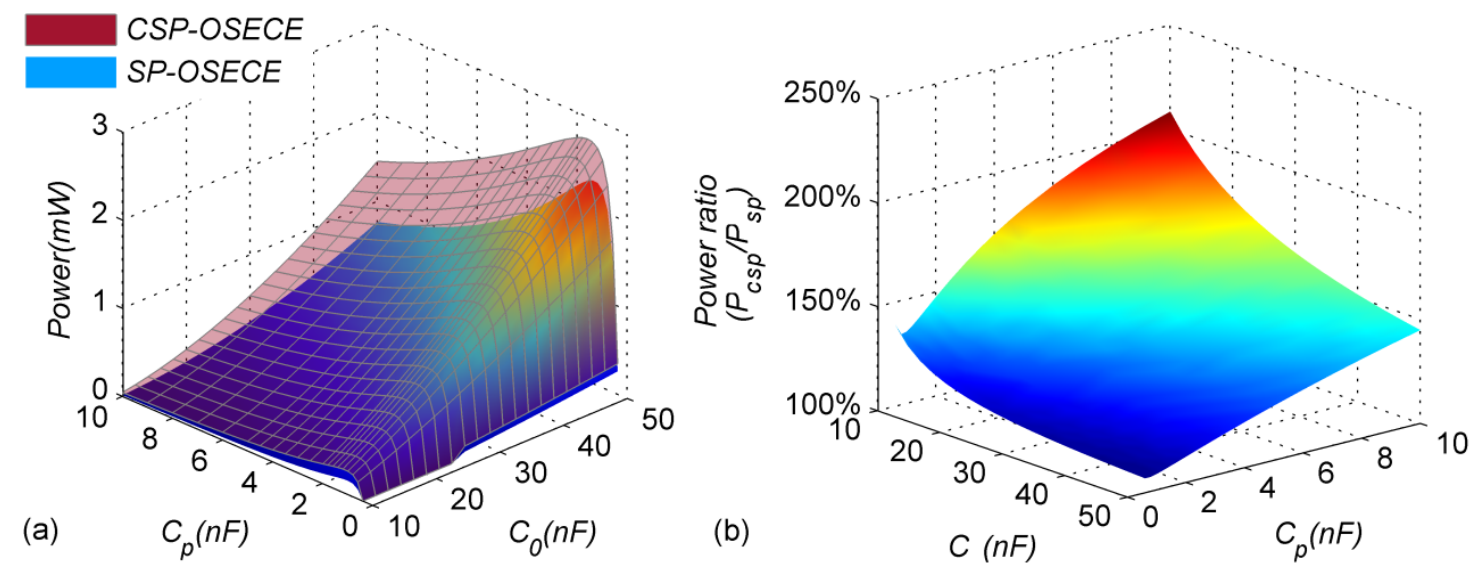

Fig. 8. (a) Power comparison of both circuits for different $C_{0}$ and $C_{p}$; (b) Power improvement of CSPOSECE with SP-OSECE as the reference.

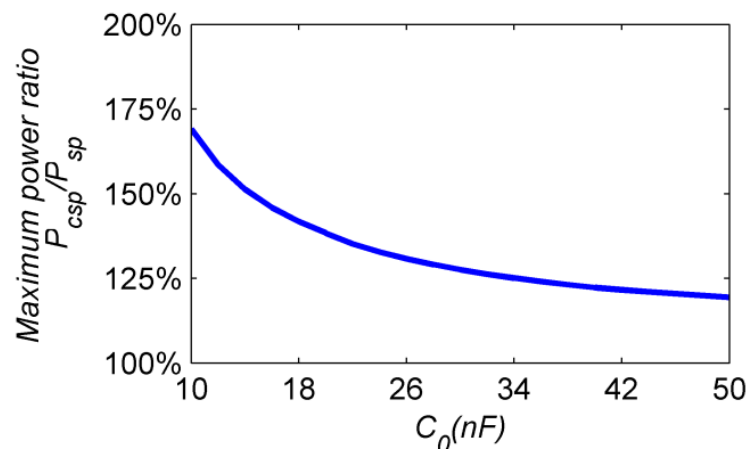

Fig. 9. Max power enhancement of CSP-OSECE versus $C_{0}$ at the optimal $C_{p}$ points with SP-OSECE as reference.

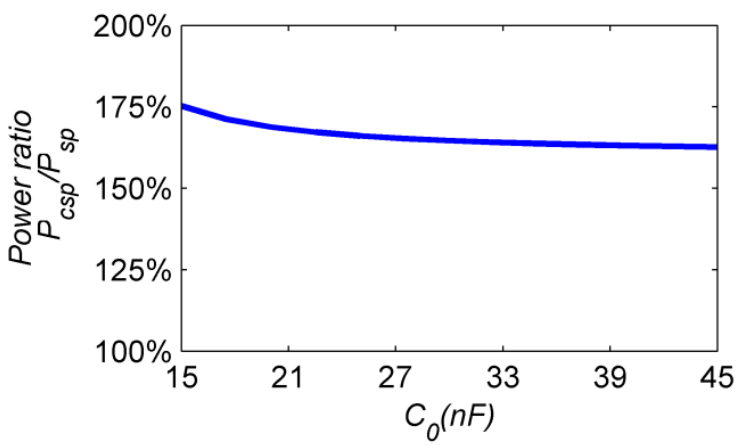

Fig. 10. Power improvement of CSP-OSECE versus $V_{o c, o r g}$ in comparison with SP-OSECE.

\section{6- Conclusion}

In this technical note, the CSP-OSECE circuit is newly proposed. By the multi-purpose use of the comparators, the specific envelope diodes can be saved. More important, a single envelope capacitor is multiplexed for both positive and negative electronic breakers instead of the two envelope capacitors in 
the regular SP-OSECE circuit. Therefore, the parasitic capacitance in the self-powered circuit is reduced to half for CSP-OSECE. It brings two benefits: (1) the available open-circuit voltage magnitude is enhanced; (2) the neutralization charge after inversion is reduced. Experimental and theoretical investigations validates that the CSP-OSECE design brings much better performance than the regular SPOSECE circuit. The advantages are especially obvious for small $C_{0}$ or large $C_{p}$ cases. It can be then concluded that the CSP-OSECE circuit is a better choice with enhanced performance and saved costs. Moreover, the compact design here can be extended to other self-powered circuits with electronic breakers. However, it is reminded that the results are only obtained for the self-powered circuit with complementary branches. In comparison with the self-powered circuit with two identical branches, the CSP-OSECE circuit shows the same advantage of less parasitic capacitance. When the switch influence is assumed the same in both configurations, it can be inferred that better power performance is still expected for CSPOSECE. As for other self-powered architectures, such as the nano-power comparator LTC1540, the performance comparison result is hard to reach directly and specific investigations are required.

\section{Acknowledgement}

The work is supported by the NSFC (National Science Foundation of China) under grant No. 51505395, the Fundamental Research Funds for the Central Universities of China (No. 2682017CX26, 2682017CY05) and the Postdoctoral Science Foundation of China (2017T100709, 2016M600747).

\section{References}

[1] Kuo C, Lin S and Wu W 2016 Fabrication and performance evaluation of a metal-based bimorph piezoelectric MEMS generator for vibration energy harvesting Smart Mater Struct. 25(10) 105016.

[2] Liu W, Liu C, Ren B, Zhu Q, Hu G and Yang W 2016 Bandwidth increasing mechanism by introducing a curve fixture to the cantilever generator. Appl Phys Lett. 109 43905. doi:10.1063/1.4960147.

[3] Liu W, Formosa F, Badel A, Agbossou A and Hu G 2016 Investigation of a buckled beam generator with elastic clamp boundary Smart Mater Struct. 25(11) 115045. doi:10.1088/0964-1726/25/11/115045.

[4] Xu J and Tang J 2015 Multi-directional energy harvesting by piezoelectric cantilever-pendulum with internal resonance Appl Phys Lett. 107 213902. doi:10.1063/1.4936607.

[5] Tao K, Tang L, Wu J, Lye S W, Chang H and Miao J 2018 Investigation of Multimodal Electret-based MEMS Energy Harvester with Impact-induced Nonlinearity J. Microelectromech. Syst. DOI: 10.1109/JMEMS.2018.2792686

[6] Ottman G K, Hofmann H F, Bhatt A C and Lesieutre G A 2002 Adaptive piezoelectric energy harvesting circuit for wireless remote power supply IEEE Trans. Power Electron. 17(5) 669-676.

[7] Guyomar D, Badel A, Lefeuvre E and Richard C 2005 Toward Energy Harvesting Using Active Materials and Conversion Improvement by Nonlinear Processing IEEE Trans. Ultrason. Ferroelectr. Freq. Control 52(4) 584. 
[8] Garbuio L, Lallart M, Guyomar D, Richard C and Audigier D 2009 Mechanical Energy Harvester With Ultralow Threshold Rectification Based on SSHI Nonlinear Technique IEEE Trans. Ind. Electron. 56(4) 1048-1056.

[9] Lallart M, Garbuio L, Petit L, Richard C and Guyomar D 2008 Double synchronized switch harvesting (DSSH): A new energy harvesting scheme for efficient energy extraction IEEE Trans. Ultrason. Ferroelectr. Freq. Control 55(10) 2119-2130.

[10] Shen H, Qiu J, Ji H, Zhu K and Balsi M 2010 Enhanced synchronized switch harvesting: a new energy harvesting scheme for efficient energy extraction Smart Mater. Struct. 19(11) 115017.

[11] Lefeuvre E, Badel A, Richard C and Guyomar D 2005 Piezoelectric Energy Harvesting Device Optimization by Synchronous Electric Charge Extraction J. Intell. Mater. Syst. Struct. 16(10) 865-876.

[12] Wu Y, Badel A, Formosa F, Liu W and Agbossou A E 2012 Piezoelectric vibration energy harvesting by optimized synchronous electric charge extraction J. Intell. Mater. Syst. Struct. 24(12) 1445-1458.

[13] Lefeuvre E, Badel A, Brenes A, Seok S, Woytasik M and Yoo C S 2017 Analysis of piezoelectric energy harvesting system with tunable SECE interface Smart Mater. Struct. 26(3).

[14] Wu Y, Badel A, Formosa F, Liu W and Agbossou A 2014 Nonlinear vibration energy harvesting device integrating mechanical stoppers used as synchronous mechanical switches J. Intell. Mater. Syst. Struct. 25(14) $1658-1663$.

[15] Liu W, Formosa F, Badel A, Wu Y and Agbossou A 2014 Self-powered nonlinear harvesting circuit with a mechanical switch structure for a bistable generator with stoppers Sensors Actuators A Phys. 216 106-115.

[16] Liu H, Liang J and Ge C 2015 A mechatronic power boosting design for piezoelectric generators Appl. Phys. Lett. 107(14) 141902.

[17] Shih Y S, Vasic D and Wu W J 2016 A non-contact mechanical solution for implementing synchronized switching techniques for energy harvesting using reed switches Smart Mater. Struct. 25(12) 125013.

[18] Chen Y Y, Vasic D, Costa F, Wu W J and Lee C K 2012 A self-powered switching circuit for piezoelectric energy harvesting with velocity control Eur. Phys. J. Appl. Phys. 57(3) 30903.

[19] Lu S and Boussaid F 2015 A Highly Efficient P-SSHI Rectifier for Piezoelectric Energy Harvesting IEEE Trans. Power Electron. 30(10) 5364-5369.

[20] Boisseau S, Gasnier P, Gallardo M and Despesse G 2013 Self-starting power management circuits for piezoelectric and electret-based electrostatic mechanical energy harvesters J. Phys. Conf. Ser. 47612080.

[21] Lallart M and Guyomar D 2008 An optimized self-powered switching circuit for non-linear energy harvesting with low voltage output Smart Mater. Struct. 17(3) 35030.

[22] Liang J and Liao W 2012 Improved design and analysis of self-powered synchronized switch interface circuit for piezoelectric energy harvesting systems IEEE Trans Ind Electron. 59(4)1950-1960.

[23] Wu Y, Badel A, Formosa F, Liu W and Agbossou A 2014 Self-powered optimized synchronous electric charge extraction circuit for piezoelectric energy harvesting J. Intell. Mater. Syst. Struct. 25(17) 2165-2176.

[24] Shi G, Xia Y, Ye Y, Qian L and Li Q 2016 An efficient self-powered synchronous electric charge extraction interface circuit for piezoelectric energy harvesting systems J Intell Mater Syst Struct. 27(16) 2160-2178. 
[25] Liu W Q, Badel A, Formosa F, Zhu Q, Zhao C and Hu G 2018 A Comprehensive Analysis and Modeling of the Self-powered Synchronous Switching Harvesting Circuit with Electronic Breakers IEEE Trans. Ind. Electron. 65(5) 3899-3909. doi: 10.1109/TIE.2017.2762640.

[26] Eltamaly A and Addoweesh K 2017 A Novel Self Power SSHI Circuit for Piezoelectric Energy Harvester IEEE Trans. Power Electron. 32(10) 7663. doi:10.1109/TPEL.2016.2636903.

[27] Lallart M 2017 Nonlinear technique and self-powered circuit for efficient piezoelectric energy harvesting under unloaded cases Energy Conversion and Management 133 444-457. doi:10.1016/j.enconman.2016.10.065. 